\title{
Electric-Magnetic Duality Invariant Lagrangians
}

\author{
Machiko Hatsuda, Kiyoshi Kamimura ${ }^{\dagger}$ and Sayaka Sekiya ${ }^{\dagger}$ \\ Theory Division, KEK, Tsukuba, Ibaraki, 305-0801, Japan \\ ${ }^{\dagger}$ Department of Physics, Toho University, Funabashi 274-8510, Japan
}

\begin{abstract}
We find general non-linear lagrangians invariant under the electric-magnetic duality. They are characterized by an arbitrary function and are reduced to the Maxwell theory in weak field limit. We present some explicit examples which include generalizations of the Born-Infeld theory.
\end{abstract}

PACS: 03.50.De, 11.10.Ef, $12.40 . \mathrm{N}$

Keywords: electric-magnetic duality, Born-Infeld theory

June, 1999

KEK-TH-631

TOHO-FP-9963

\footnotetext{
${ }^{1}$ mhatsuda@ccthmail.kek.jp, kamimura@ph.sci.toho-u.ac.jp, sekiya@ph.sci.toho-u.ac.jp.
} 


\section{Introduction}

In examining non-perturbative aspects of various theories dualities play important roles. The Montonen-Olive duality interchanges the electric charge $(e)$ and the magnetic charge $(q=n / e)$ relating the strong and the weak couplings [1]. Seiberg and Witten showed that it holds exactly at the quantum level due to the supersymmetries. It is crucial to make the models solvable [2]. This duality can be lifted to a $\operatorname{SL}(2, \mathrm{Z})$ duality by taking into account of the gauge coupling and the theta angle [3]. There is a similar duality in string theories called S-duality which interchanges the strong and the weak string couplings. It can be also lifted to a $\operatorname{SL}(2, \mathrm{Z})$ duality $\llbracket$. The S-duality is reduced to the electric-magnetic duality in 4 dimensional theories [5, 6]. It makes D3-branes self-dual [7, 8].

Among these dualities the most fundamental and familiar one is the electric-magnetic (EM) duality. The EM duality is a symmetry of free Maxwell equations under rotations of the electric and the magnetic fields in $\mathrm{U}(1)$ gauge theories. It is a symmetry between the equations of motion and the Bianchi identities. Although the duality rotations in terms of the electric and the magnetic fields are not invariance of the action they can be realized as transformations of the gauge fields and become a symmetry of the action [9, 10]. The EM duality can be lifted to a $\operatorname{SL}(2, \mathrm{R})$ duality [11, 12].

After discovery of D-branes [13] in string theories much attention is payed on the Born-Infeld action. The Born-Infeld action is one of examples of the EM duality invariant actions. The duality condition is examined as $\operatorname{Sp}(2 \mathrm{n}, \mathrm{R})$ invariance for $n \mathrm{U}(1)$ gauge fields coupled with scalars [14. In manifest Lorentz covariant approaches it is shown that there exist families of lagrangians with the duality invariance [15][16]. However closed forms, beyond the Born-Infeld, have not seem to be found so far.f

There is an alternative approach in which the EM duality invariance is manifest [17, 18. In addition to the $\mathrm{U}(1)$ gauge field, its dual gauge field is introduced. In this approach duality transformations are rotations of these gauge fields and the invariance are easily implemented. However the manifest Lorentz invariance is sacrificed for the manifest duality. A manifest Lorentz covariant version of this approach has been developed for the Maxwell theory[19].

In this paper we first review the conditions on lagrangians both in the manifest Lorentz and in the duality invariant approaches. They are reduced to a non-linear differential equation. It has general solutions characterized by an arbitrary function of one variable, $F(u)$. Thus general forms of lagrangians are found by some algebraic tasks in both approaches. They have the weak field limit to the Maxwell theory. A constant choice of $F(u)$ gives the Born-Infeld theory. We present explicit examples some of which are one-parameter families of generalized Born-Infeld actions and others is not Born-Infeld type. They do not have simple forms and may not be practical. An advantage of our formulation is that we can write equivalent lagrangians in alternative forms using with auxiliary variables. They are much simpler and useful for generalization.

\footnotetext{
2 [15] gives an example that does not have Maxwell weak field limit.
} 
In next section we give a brief review of the duality conditions and show that they are reduced to a simple non-linear differential equation. In section 3 we solve it and establish a prescription to find invariant lagrangians. It is applied to find some explicit examples in section 4. In section 5 we show forms of general invariant lagrangians with auxiliary variables. Summary and discussions are given in last section.

\section{Duality Invariance and Lorentz Invariance}

The EM duality is an invariance under $\mathrm{SO}(2)$ rotations of $\mathrm{U}(1)$ field strength $F_{\mu \nu}$ and its dual $K_{\mu \nu}$ defined by

$$
* K^{\mu \nu}=\frac{\partial L}{\partial F_{\mu \nu}}
$$

In this paper we consider pure $\mathrm{U}(1)$ gauge theories without matter field for simplicity. The infinitesimal duality transformation is

$$
\delta F=\lambda K, \quad \delta K=-\lambda F .
$$

Since a set of the field equation $\partial_{\mu} * K^{\mu \nu}=0$ and the Bianchi identity $\partial_{\mu} * F^{\mu \nu}=0$ is invariant under it the duality condition is the invariance of the defining relation of $K$ in (2.2). It has been examined in general matter coupling theories [14]. The compatibility condition of the duality rotations with the definition (2.2) is

$$
F * F+K * K=0 .
$$

Although it does not mean the invariance of the action it can be shown that (2.3) indeed becomes a symmetry transformation of the action when it is interpreted as a transformation of the $\mathrm{U}(1)$ gauge fields [9, 10].

The Gaillard-Zumino condition (2.4) is expressed as a differential equation for lagrangians. In the Lorentz covariant formulation lagrangians are assumed as functions of $F F$ and $F * F$,

$$
L=L(t, z), \quad t \equiv \frac{1}{4} F_{\mu \nu} F^{\mu \nu}, \quad z \equiv \frac{1}{4} F_{\mu \nu} * F^{\mu \nu}
$$

Using (2.2) the dual field strength is

$$
* K=L_{t} F+L_{z} * F, \rightarrow K=-L_{t} * F+L_{z} F
$$

\footnotetext{
${ }^{3}$ Our conventions are

$$
* F^{\mu \nu} \equiv \frac{1}{2} \epsilon^{\mu \nu \rho \sigma} F_{\rho \sigma}, \quad \frac{\partial F_{\rho \sigma}}{\partial F_{\mu \nu}}=\delta_{\rho}^{\mu} \delta_{\sigma}^{\nu}-\delta_{\sigma}^{\mu} \delta_{\rho}^{\nu}, \quad \epsilon^{0123}=1, \quad \eta_{\mu \nu}=\{-;+,+,+\} .
$$
}


where $L_{t}=\frac{\partial L}{\partial t}$, etc. and the Gaillard-Zumino condition (2.4) is expressed as 16]

$$
z L_{t}^{2}-2 t L_{z} L_{t}-z L_{z}^{2}-z=0
$$

The lagrangians of the Maxwell and the Born-Infeld theories are

$$
L^{(M)}=-t, \quad L^{(B I)}=1-\sqrt{1+2 t-z^{2}}
$$

which are solutions of (2.7) respectively.

In the above Lorentz invariant approach the EM duality is not manifest. There is an alternative approach in which the duality invariance is manifest. In addition to the $\mathrm{U}(1)$ field $A_{\mu}^{(1)}$ a dual gauge field $A_{\mu}^{(2)}$ is introduced. The duality transformation is realized as $\mathrm{SO}(2)$ rotation mixing two gauge fields. In this form the duality can be satisfied manifestly while the Lorentz invariance is not. The Schwarz-Sen lagrangian [17] for the Maxwell theory is generalized as [18]

$$
L^{S S}=\frac{1}{2} \mathcal{L}^{\alpha \beta} B^{i(\alpha)} E_{i}^{(\beta)}-H,
$$

where $E_{i}^{(\alpha)}=F_{0 j}^{(\alpha)}, B^{i(\alpha)}=\frac{1}{2} \epsilon^{i j k} F_{j k}^{(\alpha)}$ and $\mathcal{L}^{12}=-\mathcal{L}^{21}=1 . \quad H$ is a function of $B^{i(\alpha)}$ and is invariant under $\mathrm{SO}(2)$ rotations of the two gauge potentials (with respect to the indices $(\alpha=1,2))$ and spatial 3D rotations (with respect to the indices $(i=1,2,3))$. It is a function of $t$ and $y$ defined by

$$
t=\frac{1}{2}\left(\left(B^{(1)}\right)^{2}+\left(B^{(2)}\right)^{2}\right), \quad y=\frac{1}{2}\left(\epsilon^{i j k} B_{j}^{(1)} B_{k}^{(2)}\right)^{2} .
$$

and plays a role of (non-vanishing part of) the Hamiltonian in the canonical formalism.

Variation of the action with respect to $A_{0}^{(\alpha)}$ gives identities

$$
\partial_{i} B^{i(\alpha)}=0, \quad(\alpha=1,2)
$$

Using this redundancy of $A_{0}^{(\alpha)}$ equations of motion of $A_{i}^{(\alpha)}$

$$
\epsilon^{i j k} \partial_{j}\left(\mathcal{L}^{\alpha \beta} E_{k}^{(\beta)}-\frac{\partial H}{\partial B^{k(\alpha)}}\right)=0
$$

can be reduced to

$$
\left(\mathcal{L}^{\alpha \beta} E_{k}^{(\beta)}-\frac{\partial H}{\partial B^{k(\alpha)}}\right)=0 .
$$

They are relations between $E^{(\alpha)}$ 's and $B^{(\alpha)}$ 's and (generalized) Maxwell equations follow from the Bianchi identities $\partial * F^{(\alpha)}=0$.

The lagrangian (2.9) does not possess manifest Lorentz invariance. It is guaranteed by covariance of the equations of motion (2.13) under Lorentz boosts,

$$
\delta E_{i}^{(\alpha)}=-\epsilon_{i j k} w^{j} B^{k(\alpha)}, \quad \delta B^{i(\alpha)}=\epsilon^{i j k} w_{j} E_{k}^{(\alpha)} .
$$


The condition is expressed as a differential equation for $H(t, y)$,

$$
H_{t}^{2}+2 t H_{t} H_{y}+2 y H_{y}^{2}-1=0 .
$$

It has also been derived from the conservation of energy momentum tensors [18]. $H$ for the Maxwell and the Born-Infeld theories are

$$
H^{(M)}=t, \quad H^{(B I)}=\sqrt{1+2 t+2 y}-1 .
$$

We have obtained two differential equations, (2.7) in the manifest Lorentz invariant formalism and (2.15) in the manifest duality invariant formalism. They are converted to each other by a change of variables

$$
y=-\frac{1}{2} z^{2}
$$

and

$$
L(t, z)=-H(t, y)
$$

It is noted the physical region of the variable $y$ in (2.10) is positive while the relation (2.17) requires continuation of $y$ to negative region. It is true for $t$ 's in (2.5) and (2.10).

A purpose of this paper is to find invariant lagrangians by solving (2.7) and/or (2.15). They are further simplified by introducing new variable $x$

$$
x=\sqrt{t^{2}+z^{2}}=\sqrt{t^{2}-2 y}
$$

and

$$
L(t, z)=-H(t, y)=k(t, x) .
$$

In terms of new function $k(t, x)$ both differential equations (2.7) and (2.15) become

$$
k_{t}^{2}-k_{x}^{2}-1=0 .
$$

This differential equation has been obtained using geometrical consideration in [15]. In terms of the function $k(t, x)$ the Maxwell and Born-Infeld lagrangians are

$$
k^{(M)}=-t, \quad k^{(B I)}=1-\sqrt{(1+t)^{2}-x^{2}} .
$$

\footnotetext{
${ }^{4}$ The signs of $L$ and $H$ are not determined from (2.7) and 2.15$)$. They are chosen by convention.
} 


\section{General Solutions}

A form of general solutions of the differential equation (2.21) have been discussed in [16] and a prescription to obtain invariant lagrangians was presented. In this section we give an alternative form of general solutions.

We solve the differential equation (2.21) by setting $k_{t}=-\cosh \lambda, k_{x}=\sinh \lambda$ and $\tanh \lambda=u$,

$$
k_{t}=\frac{-1}{\sqrt{1-u(t, x)^{2}}}, \quad k_{x}=\frac{u(t, x)}{\sqrt{1-u(t, x)^{2}}} .
$$

Here minus sign of $k_{t}$ is chosen for the Maxwell limit (see (2.22)). They verify (2.21) trivially while it requires an integrability condition

$$
u_{t}(t, x)+u(t, x) u_{x}(t, x)=0 .
$$

This is a familiar non-linear equation and general solutions are obtained by solving arbitrary implicit function of two variables, $u$ and $x-t u$, for $u$ [20]. Let $G(u)$ be an arbitrary function of $u$ and solve

$$
x-t u=G(u)
$$

for $u$. The $u(t, x)$ is a solution of (3.2) and $k(t, x)$ is found by integrating (3.1). The $k(t, x)$ obtained in this procedure solves (2.21) and the corresponding duality invariant lagrangians are found through (2.20). We can prove (3.2) by taking derivatives of (3.3) with respect to $t$ and $x$

$$
u_{t}=\frac{-u}{t+G^{\prime}(u)}, \quad u_{x}=\frac{1}{t+G^{\prime}(u)}
$$

The arbitrariness of $G(u)$ is understood by regarding (3.2) as a t-evolution equation. At time $t=0$ the initial data $u(0, x) \equiv G^{-1}(x)$ is taken arbitrarily. The $u(t, x)$ at later time is determined as long as differentiability is maintained.

For a given $G(u)$ suppose (3.3) is solved for $u$. In order to integrate (3.1) it is convenient to make change of the variables

$$
(t, x) \rightarrow(t, u), \quad \tilde{k}(t, u) \equiv k(t, x) .
$$

Using (3.1) and (3.4)

$$
\tilde{k}_{t}(t, u)=-\sqrt{1-u^{2}}, \quad \tilde{k}_{u}(t, u)=\frac{u\left(t+G^{\prime}(u)\right)}{\sqrt{1-u^{2}}},
$$

whose integrability is guaranteed by (3.3). The first equation in (3.6) is integrated with respect to $t$

$$
\tilde{k}(t, u)=-t \sqrt{1-u^{2}}+g(u)
$$


and $g^{\prime}(u)$ is determined by the second equation in (3.6),

$$
g^{\prime}(u)=\frac{u G^{\prime}(u)}{\sqrt{1-u^{2}}}
$$

It may be integrated by changing the integration variable from $u$ to $\tilde{u}=\sqrt{1-u^{2}}$

$$
g(u)=\int d \tilde{u}\left\{-G^{\prime}\left(\sqrt{1-\tilde{u}^{2}}\right)\right\}+\text { const. }
$$

We can summarize our result as follows. Since $G(u)$ is arbitrary we start choosing an arbitrary function $F(u) \equiv G^{\prime}(u) . G(u)$ and $\tilde{k}(t, u)$ are determined in terms of $F(u)$ as

$$
G(u)=\int_{0}^{u} d \tilde{u} F(\tilde{u})+c_{1}
$$

and

$$
\tilde{k}(t, u)=-t \sqrt{1-u^{2}}-\int_{1}^{\sqrt{1-u^{2}}} d \tilde{u} F\left(\sqrt{1-\tilde{u}^{2}}\right)+c_{2} .
$$

The solution $k(t, x)$ of (2.21) is determined by solving (3.3) for $u$ with $G(u)$ given by (3.10).

Two integration constants in (3.10) and (3.11) are determined if we impose a condition that the system goes to the Maxwell theory in the weak field limit. Since the Maxwell theory is described by $k(t, x)$ in $(2.22)$,

$$
k^{(M)}(t, x)=-t, \quad \rightarrow \quad k_{t}=-1 \quad \rightarrow \quad u=0
$$

then (3.3) for the weak field $t=x=0$ must have the solution $u=0$. This condition determines the integration constant $c_{1}$ in (3.10) and $c_{2}$ in (3.11) as

$$
G(0)=c_{1}=0, \quad c_{2}=0
$$

\section{Examples}

For a given function $F(u)$ we can in principle find the corresponding lagrangians, manifest Lorentz invariant $L(t, z)$ in (2.5) and manifest duality invariant $L^{S S}$ in (2.9). However there are two obstructions practically. One is to solve (3.3) algebraically for $u$. Another is to integrate (3.10) and (3.11) explicitly. There are some limitations on the arbitrary function $F(u)$ to get closed forms of lagrangians. The situation is same in the method by Gaillard and Zumino [16] and they didn't give explicit example beyond the Born-Infeld lagrangian. In this section we present some examples whose lagrangians are found in closed forms. 


\subsection{Maxwell and Born-Infeld theories}

The simplest choice of $F(u)$ may be a constant,

$$
F(u)=\frac{1}{b}
$$

From (3.10) and (3.11) $G(u)$ and $g(u)$ are determined as

$$
\begin{aligned}
G(u) & =\int_{0}^{u} d \tilde{u} F(\tilde{u})=\frac{u}{b} . \\
g(u) & =-\int_{1}^{\sqrt{1-u^{2}}} d \tilde{u} F\left(\sqrt{1-\tilde{u}^{2}}\right)=\frac{1-\sqrt{1-u^{2}}}{b} .
\end{aligned}
$$

(3.3) is solved for $u$ as

$$
G(u)=\frac{u}{b}=x-t u, \quad \rightarrow \quad u=\frac{b x}{1+b t} .
$$

Then

$$
\tilde{k}(t, u)=-t \sqrt{1-u^{2}}+\frac{1-\sqrt{1-u^{2}}}{b}
$$

and

$$
k(t, x)=\frac{1}{b}\left(1-\sqrt{(1+b t)^{2}-b^{2} x^{2}}\right) .
$$

The lagrangian is given in terms of $t=\frac{1}{4} F F$ and $z=\frac{1}{4} F * F$ as

$$
L^{(B I)}(t, z)=k\left(t, \sqrt{t^{2}+z^{2}}\right)=\frac{1}{b}\left(1-\sqrt{1+2 b t-b^{2} z^{2}}\right) .
$$

This is the Born-Infeld lagrangian ( the constant $b$ is taken to be unity by rescaling of space-time coordinates and $A_{\mu}$ as long as $b$ is finite). In the weak field expansion it is

$$
k^{(B I)}(t, x) \sim-t+\frac{b}{2} x^{2}-\frac{b^{2}}{2} t x^{2}+\frac{b^{3}}{8} x^{2}\left(4 t^{2}+x^{2}\right)-\ldots
$$

and the Maxwell theory is given in $b \rightarrow 0$ limit

$$
L^{(M)}(t, z)=k^{(M)}(t, x)=-t .
$$

\subsection{Second Example}

Next we choose $F(u)$ as a linear function of $u$,

$$
F(u)=\frac{1+2 a u}{b}
$$


where $a$ and $b$ are constants. From (3.10) and (3.11)

$$
\begin{aligned}
G(u) & =\frac{u}{b}(1+a u) \\
g(u) & =\left(\frac{1}{b}+\frac{a \pi}{2 b}\right)-\frac{1}{b}(1+a u) \sqrt{1-u^{2}}-\frac{a}{b} \cos ^{-1} u .
\end{aligned}
$$

(3.3) determines $u(t, x)$

$$
G(u)=\frac{a u^{2}+u}{b}=x-t u, \rightarrow u=\frac{b x}{1+b t} e,
$$

where

$$
e=\frac{2}{1+\sqrt{1+\frac{4 a b x}{(1+b t)^{2}}}}
$$

$e(t, x)$ goes to 1 as $a \rightarrow 0$. Then

$$
\begin{aligned}
k(t, x)= & \frac{1}{b}\left(1-\sqrt{(1+b t)^{2}-b^{2} x^{2} e^{2}}\right) \\
& +\frac{a}{b}\left(\frac{\pi}{2}-\left(\frac{b x e}{1+b t}\right) \sqrt{1-\left(\frac{b x e}{1+b t}\right)^{2}}-\cos ^{-1}\left(\frac{b x e}{1+b t}\right)\right) .
\end{aligned}
$$

We can consider two limiting cases. The $a \rightarrow 0$ limit naturally goes back to the BornInfeld lagrangian that is expected from the form of $F(u)$ in (4.10). The small $b$ expansion is the weak field expansion

$$
k \sim-t+\frac{b}{2} x^{2}-\frac{b^{2}}{2} x^{2}\left(t+\frac{2 a}{3} x\right)+\frac{b^{3}}{8} x^{2}\left\{\left(4 t^{2}+x^{2}\right)+8 a t x+4 a^{2} x^{2}\right\}+\ldots
$$

which is compared to the Born-Infeld case in (4.8).

\section{$4.3 \quad$ Example 3}

In the previous example an infinite limit case of $a$ must be treated separately and provides a qualitatively different model. We choose $F(u)$ as

$$
F(u)=\frac{u}{b}
$$

where $b$ is a constant. From (3.10) and (3.11)

$$
G(u)=\frac{u^{2}}{2 b}, \quad g(u)=-\frac{1}{2 b}\left(u \sqrt{1-u^{2}}+\cos ^{-1} u-\frac{\pi}{2}\right) .
$$


$u(t, x)$ is determined as

$$
G(u)=\frac{u^{2}}{2 b}=x-t u, \quad \rightarrow \quad u(t, x)=\sqrt{b^{2} t^{2}+2 b x}-b t
$$

and

$$
\begin{aligned}
k(t, x)= & -\frac{1}{2 b}\left(\sqrt{b^{2} t^{2}+2 b x}+b t\right) \sqrt{1-\left(\sqrt{b^{2} t^{2}+2 b x}-b t\right)^{2}} \\
& +\frac{1}{2 b}\left(\frac{\pi}{2}-\cos ^{-1}\left(\sqrt{b^{2} t^{2}+2 b x}-b t\right)\right) .
\end{aligned}
$$

It has the weak field expansion

$$
k \sim-t+\frac{2 \sqrt{2} b^{\frac{1}{2}}}{3} x^{\frac{3}{2}}-b t x+\frac{b^{\frac{3}{2}}}{5 \sqrt{2}} x^{\frac{1}{2}}\left(5 t^{2}+4 x^{2}\right)-\frac{b^{2}}{6} t\left(2 t^{2}+9 x^{2}\right)+\ldots,
$$

which is a power series of $b^{\frac{1}{2}}$, half integral power of $t$ and $x$, and is quite different from the Born-Infeld type.

\subsection{Example 4,5}

Finally we give two examples. There appears no trigonometric functions in the lagrangians. They also give generalizations of the Born-Infeld action.

One is given by choosing $F(u)$ as

$$
F(u)=\frac{1+a u^{2}}{b}
$$

and

$$
\begin{aligned}
& k(t, x)=\frac{1}{b}\left(1-\sqrt{(1+b t)^{2}-b^{2} x^{2} e^{2}}\right) \\
&+\frac{2 a}{3 b}\left(1-\sqrt{(1+b t)^{2}-b^{2} x^{2} e^{2}} \frac{\left((1+b t)^{2}+\frac{b^{2} x^{2} e^{2}}{2}\right)}{(1+b t)^{3}}\right), \\
& e(t, x) \equiv \frac{3}{e_{0}^{\frac{2}{3}}+1+e_{0}^{-\frac{2}{3}}}, \quad e_{0}(t, x) \equiv \sqrt{1+\frac{9 a b^{2} x^{2}}{4(1+b t)^{3}}}+\sqrt{\frac{9 a b^{2} x^{2}}{4(1+b t)^{3}}},
\end{aligned}
$$

where $e(t, x)$ and $e_{0}(t, x)$ are functions whose values are 1 when $a=0$. In the weak field limit (4.23) is,

$$
k \sim-t+\frac{b}{2} x^{2}-\frac{b^{2}}{2} t x^{2}+\frac{b^{3}}{8} x^{2}\left(\left(4 t^{2}+x^{2}\right)-\frac{2}{3} a x^{2}\right)+\ldots
$$


The other is given by choosing $F(u)$ as

$$
F(u)=\frac{1+a\left(3 u-4 u^{3}\right)}{b} .
$$

The coefficients of $u$ and $u^{3}$ terms are adjusted so that the arcsine term does not appear in $k$. I The result is

$$
k(t, x)=\frac{1}{b}\left(1-\sqrt{(1+b t)^{2}-b^{2} x^{2} e^{2}}\left(1-\frac{a b^{3} x^{3} e^{3}}{(1+b t)^{4}}\right)\right) .
$$

Here $e(t, x)$ is a solution of forth order equation

$$
1-e-\frac{3 a b x}{2(1+b t)^{2}} e^{2}+\frac{a b^{3} x^{3}}{(1+b t)^{4}} e^{4}=0
$$

which goes to 1 when $a=0$.

For weak fields it is

$$
k \sim-t+\frac{b}{2} x^{2}-\frac{b^{2}}{2} x^{2}(t+a x)+\frac{b^{3}}{8} x^{2}\left(\left(4 t^{2}+x^{2}\right)+12 a t x+9 a^{2} x^{2}\right)+\ldots
$$

\section{Lagrangians with an Auxiliary Variable}

In previous section we presented several examples in which their lagrangians are given in closed forms. As was seen they are not simple enough for practical applications. It mainly came from the necessity of solving the equation for $u,(3.3)$,

$$
x-t u=G(u) .
$$

In this section we propose an alternative formulation in which this equation is treated as a constraint. We construct a lagrangian that is equivalent, at least classically, to (3.11) and (3.3),

$$
L(u, t, x)=-\frac{t-u x}{\sqrt{1-u^{2}}}-\int_{0}^{u} \frac{G(r)}{\left(1-r^{2}\right)^{\frac{3}{2}}} d r .
$$

Here $u$ is regarded as an independent variable and $G(u)$ is the arbitrary function satisfying $G(0)=0$. By taking a variation of the lagrangian with respect to $u$ we obtain (5.1) as an equation of motion. If it would be solved for $u$ and the solution is used in the lagrangian

\footnotetext{
${ }^{5}$ If the constant term of $F(u)$ in $(\sqrt{4.26})$ is absent it givs a non-Born-Infeld type model as example 3.
} 
(5.2), $L(u(t, x), t, x)$ becomes $k(t, x)$ in (3.11). Actually the lagrangian (5.2) is $\tilde{k}(t, u)$ of (3.7) in which $g(u)$ is replaced as follows,

$$
\begin{aligned}
g(u)=\int_{0}^{u} d r \frac{r G^{\prime}(r)}{\sqrt{1-r^{2}}} & =\frac{u G(u)}{\sqrt{1-u^{2}}}-\int_{0}^{u} d r G(r)\left(\frac{r}{\sqrt{1-r^{2}}}\right)^{\prime} \\
& \rightarrow \frac{u(x-u t)}{\sqrt{1-u^{2}}}-\int_{0}^{u} d r \frac{G(r)}{\left(1-r^{2}\right)^{\frac{3}{2}}} .
\end{aligned}
$$

Here $G(u)$ is replaced by $(x-u t)$ only in the first term of right hand side.

The lagrangian $L(u, t, x)$ in (5.2) is often useful since the equation for $u$ (5.1) is built in the lagrangian in stead of imposing by hand. In this form it is not always necessary to solve the equation for $u$ (5.1) explicitly for many purposes. Especially in quantum theory (5.1) may be left as a constraint rather than a strong equality.

In the lagrangian (5.2) $G(u)$ is arbitrary except $G(0)=0.0$ We can regard the last term as arbitrary function $M(u)$ subject to the boundary condition

$$
M(0)=M^{\prime}(0)=0
$$

and the general form of duality invariant lagrangian is written as

$$
L(u, t, x)=-\frac{t-u x}{\sqrt{1-u^{2}}}+M(u) .
$$

Or equivalently it is expressed, using $u=\tanh \lambda$, as

$$
\tilde{L}(\lambda, t, x)=-t \cosh \lambda+x \sinh \lambda+\tilde{M}(\lambda), \quad \tilde{M}(0)=\tilde{M}^{\prime}(0)=0 .
$$

For Born-Infeld case

$$
M(u)=\frac{1}{b}\left(1-\frac{1}{\sqrt{1-u^{2}}}\right), \quad \tilde{M}(\lambda)=-\frac{2}{b}\left(\sinh ^{2} \frac{\lambda}{2}\right) .
$$

Finally we list forms of the lagrangian (5.2) for the examples in the last section.

The Born-Infeld lagrangian

$$
L^{B I}(u, t, x)=\frac{1}{b}\left(1+\frac{-(1+b t)+b u x}{\sqrt{1-u^{2}}}\right) .
$$

Example 2

$$
L(u, t, x)=L^{B I}(u, t, x)+\frac{a}{b}\left(-\frac{u}{\sqrt{1-u^{2}}}+\sin ^{-1} u\right)
$$

Example 3

$$
L(u, t, x)=\frac{-2 b t-u+2 b u x}{2 b \sqrt{1-u^{2}}}+\frac{\sin ^{-1} u}{2 b} .
$$

\footnotetext{
${ }^{6}$ More correctly $G(u)=0$ gives a solution $u=0$, see the discussion of (3.12). For example a model with $G(u) \equiv 0$ gives $u=x / t$ and does not have the correct Maxwell limit.
} 
Example 4

$$
L(u, t, x)=L^{B I}(u, t, x)+\frac{2 a}{3 b}\left(1-\frac{2-u^{2}}{2 \sqrt{1-u^{2}}}\right) .
$$

Example 5

$$
L(u, t, x)=L^{B I}(u, t, x)-\frac{a u^{3}}{2 b \sqrt{1-u^{2}}} .
$$

\section{Discussions}

In this paper we have shown that the EM duality and the Lorentz invariant lagrangians can be constructed from solutions of the simple non-linear differential equation (3.2). We have obtained general solutions which are characterized by the function $F(u)$. Manifest Lorentz invariant lagrangians and manifest duality invariant lagrangians are given by (2.20). We have constructed several examples in closed forms explicitly. The lagrangians in examples 2, 4 and 5 are one-parameter families of generalized Born-Infeld theories. They go back to the Born-Infeld lagrangian for $a \rightarrow 0$ limit and have subtly different weak field expansions for finite value of $a$. The lagrangian in example 3 has different property from others and does not have the limit to the Born-Infeld theory.

In [16] Gaillard and Zumino have given a prescription to find general solutions of the differential equation (2.21). It is characterized by one arbitrary function $v(s)$ as

$$
L=\frac{2 p}{v^{\prime}(s)}+v(s), \quad t=p+q, \quad x=p-q,
$$

where $s(p, q)$ is determined by solving

$$
q=\frac{p}{\left(v^{\prime}(s)\right)^{2}}+s
$$

for $s$. The relation to ours is found by comparing (variation of) lagrangians as follows,

$$
\begin{aligned}
& x-t u=G(u) \quad q=\frac{p}{v^{\prime}(s)^{2}}+s \\
& \uparrow \quad \uparrow \\
& u=u(t, x) \quad \leftrightarrow \quad u=\frac{v^{\prime}(s)^{2}-1}{v^{\prime}(s)^{2}+1} \quad \leftrightarrow \quad s=s(p, q)
\end{aligned}
$$

This diagram relates the arbitrary functions $G(u)$ and $v^{\prime}(s)$. For the Born-Infeld theory it is

$$
G(u)=\frac{u}{b} \leftrightarrow v^{\prime}(s)=-\sqrt{\frac{1}{1+2 b s}} .
$$


For a given $k(t, x)$ we can make both manifest Lorentz invariant formulation with the lagrangian $L(t, x)$ in (2.5) and manifest duality invariant one with $L^{(S S)}$ in (2.9). It is interesting to know whether these two lagrangians are describing same system or not. Since (2.18) is simply a relation of functional forms of two lagrangians in our formalism it is not evident that they are equivalent. The equivalence is discussed in [18] that the latter is derived from the former. For the Maxwell and the Born-Infeld theories it is explicitly shown that $k$ 's in (2.22) are giving $L$ 's in (2.8) and $H$ 's in (2.16).

An advantage of our formulation is that it leads to the general form of the lagrangians

in (5.2) ( or (5.5) and (5.6) ) characterized by the arbitrary functions with its boundary conditions. They contain auxiliary variable $u$ whose equation of motion gives (5.1). It is equivalent to the lagrangian in which $u$ is solved as a function of $t$ and $x$. Using with the auxiliary variable lagrangians have relatively simple forms and much easier to be handled. These forms of lagrangians may be useful in development of general duality invariant theories.

\section{Acknowledgements}

One of the authors (KK) would like to thank Joaquim Gomis for valuable discussions. 


\section{References}

[1] C.Montonen and D.Olive, Phys. Lett. B72 (1977) 117;

P.Goddard, J.Nuyts, Nucl. Phys. B125 (1977) 1.

[2] N.Seiberg and E.Witten, Nucl. Phys. B426 (1994) 19, Erratum,ibid. B430 (1994) 485.

[3] J.Cardy and E.Rabinovici, Nucl. Phys. bf B205 (1982) 1;

J.Cardy, Nucl. Phys. B205 (1982) 17.

A.Shapere and F.Wilczek, Nucl. Phys. B320 (1989) 669.

[4] A.Font, L.Ibanez, D.Lust and F.Quevedo, Phys. Lett.B249 (1990) 35.

[5] C.Hull and P.K.Townsend, Nucl. Phys. B438 (1995) 109.

[6] E.Witten, Nucl. Phys. B443 (1995) 85.

[7] A. A. Tseytlin, Nucl. Phys.B469 (1996) 51-67.

[8] Y.Igarashi, K.Itoh and K.Kamimura, Nucl. Phys.B536 (1999) 469-482.

[9] S.Deser and C.Teitelboim, Phys. Rev. D13 (1976) 1592-1597.

[10] Y.Igarashi, K.Itoh and K.Kamimura, Nucl. Phys.B536 (1999) 454-468.

[11] G. W. Gibbons and D. A. Rasheed, Phys. Lett. B365 (1996) 46-50.

[12] M.K.Gaillard and B.Zumino, Self-Duality in Nonlinear Electromagnetism, "Supsersymmetry and quantum field theory" 121-129 (Kharkov 1997), hep-th/9705226.

[13] J.Polchinski, Phys. Rev. Lett. 75 (1995) 4724; TASI Lectures on D-Branes, hepth/9611050.

[14] M.K.Gaillard and B.Zumino, Nucl. Phys.B193 (1981) 221-244.

[15] G. W. Gibbons and D. A. Rasheed, Nucl. Phys. B454 (1995) 185-206.

[16] M.K.Gaillard and B.Zumino, Nonlinear Electromagnetic Self-Duality and Legendre Transformations, Contributed to "A Newton Institute Euroconference on Duality and Supersymmetric Theories", Cambridge, England, 7-18,Apr 1997, hep-th/9712103.

[17] J.H.Schwarz and A.Sen, Nucl. Phys.B411 (1994) 35-63.

[18] S.Deser,Ö.Sarioglu, Phys.Lett.B423 (1998) 369.

[19] P.Pasti, D.Sorokin and M.Tonin, Phys. Rev. D52 (1995) R4277-4281.

[20] For example, see G.B.Whitham "Linear and non-linear waves", (Jhon Wiley and Sons 1974). 